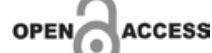

RELATO DE CASO

\title{
Câncer da Vesícula Biliar - Relato de Casos
}

Correspondence addresses: Dr. André Ney M. Freire andreney.freire@gmail.com

Received: September 18, 2021

Revised: October 28, 2021

Accepted: November 16, 2021

Published: December 28, 2021

Data Availability Statement: All relevant data are within the paper and its Supporting Information files.

Funding: This work was the result of authors' initiative. There was no support of research or publication funds.

Competing interests: The authors have declared that no competing interests exist.

\section{Copyright}

(C) 2021 by Santa Casa de Misericórdia da Bahia. All rights reserved.

ISSN: 2526-5563

e-ISSN: 2764-2089

DOI: 10.35753

\author{
Gallbladder Cancer - Case Reports
}

O câncer da vesícula biliar é uma doença que representa um grande desafio para médicos clínicos e cirurgiões, por possuir características especiais: alta capacidade de disseminação, invasão por contiguidade e continuidade, crescimento rápido e agressivo, além de apresentação clínica insidiosa, geralmente apresentando sintomas quando o quadro já está avançado. Apresentamos dois casos com desfechos completamente diferentes: um do Hospital Santa Izabel e outro do hospital especializado em câncer A.C. Camargo de São Paulo. Discutimos ainda o tratamento atual e as possíveis intervenções que podem ser traçadas no futuro para 0 aprimoramento no diagnóstico e na terapêutica dessa grave doença.

Palavras-Chave: Vesícula Biliar; Câncer; Neoplasia de Vesícula Biliar; Colecistectomía; Colelitíase.

Gallbladder cancer is a disease that represents a challenge for clinicians and surgeons, as it has some characteristics: high dissemination capacity, invasion by contiguity and continuity, rapid and aggressive growth, in addition to an insidious clinical presentation, usually presenting symptoms when the frame is already advanced. We presented two cases with different outcomes: one from the Santa Izabel Hospital and the other from the A.C. Camargo cancer hospital in São Paulo. We also discuss the current treatment and possible interventions that can improve the diagnosis and treatment in the future of this disease.

Keywords: Gallbladder; Cancer; Gallbladder Neoplasm; Cholecystectomy; Cholelithiasis.

\section{Introdução}

O câncer de vesícula biliar (CVB) é a mais frequente neoplasia das vias biliares. Contudo, ela é uma neoplasia relativamente rara. ${ }^{1}$ Sua incidência varia dependendo da região geográfica e grupos étnicos. Representa aproximadamente $3 \%$ dos tumores do trato digestório. Sua incidência varia de 0,2 a 7,1 por 100.000 entre homens e mulheres. A maioria dos CVBs são achados incidentais, sem diagnóstico prévio, em pacientes submetidos à colecistectomia por colelitíase, e ocorre em $0,25 \%$ a $3 \%$ dos casos. ${ }^{2,3}$ 
Devido às características próprias da doença e à inespecificidade do quadro clínico, o prognóstico do CVB é reservado. Estima-se que $85 \%$ dos pacientes morram um ano após terem sido diagnosticados. ${ }^{4,5}$ Isso ocorre porque, na grande maioria dos casos, o diagnóstico é realizado tardiamente em estágios avançados, o que compromete os resultados do seu tratamento com consequente aumento da morbimortalidade. ${ }^{6}$

O CVB caracteriza-se por invasão local (fígado, vias biliares e estruturas vasculares), podendo, ainda, promover metástases para linfonodos regionais e à distância, associado a uma média de sobrevida curta e uma alta taxa de recorrência local. ${ }^{3}$ Um estudo realizado no Hospital de Clínicas da Faculdade de Ciências Médicas da Universidade Estadual de Campinas - UNICAMP - mostrou que de 893 pacientes submetidos à colecistectomia, $13(1,4 \%)$ tiveram o diagnóstico de CVB. Desses, em 9 (70\%) não havia suspeita de CVB. ${ }^{7}$

\section{Relato de Casos}

Paciente, 70 anos, sexo feminino, atendida no Hospital Santa Izabel (HSI), Salvador, Bahia, cursou com dor abdominal e cólica por 32 anos, associada a flatulência. Manteve esse quadro com episódios esporádicos até 2010 quando iniciou a investigação. Em 2013 uma ultrassonografia de abdome total demonstrou a presença de dois cálculos na Vesícula Biliar (VB) medindo 20 $\mathrm{mm}$ e $22,5 \mathrm{~mm}$ de diâmetro. Posteriormente, fez uma nova ultrassonografia que demonstrou aumento no tamanho dos cálculos, sendo indicada a colecistectomia por videolaparoscopia.

Os marcadores tumorais não mostraram alterações, Ca 19.9 de 18 UI/L (Referência 35 UI/L), e o Antígeno Carcinoembriogênico 2,6 ng/ $\mathrm{mL}$ (Referência até $3 \mathrm{ng} / \mathrm{mL}$ ). A colecistectomia foi realizada no dia 12/08/2016, sem intercorrências, com boa recuperação e alta no dia seguinte. $\mathrm{O}$ exame anatomopatológico da VB diagnosticou neoplasia intraepitelial de alto grau, metaplasia intestinal e colecistite crônica calculosa.
O seguimento foi feito por médico oncologista do HSI e pela equipe de cirurgia geral. Foi indicada a realização de vários exames de imagem, incluindo ressonância magnética e retornos regulares a cada quatro meses. Após 5 anos, o último retorno em dezembro de 2021, não havia sinais de doença em atividade.

Outro paciente, 79 anos, sexo masculino, cardiopata grave, hipertenso e diabético. História breve de desconforto e distensão abdominal. Foi submetido a colecistectomia videolaparoscópica em um hospital na cidade de São Paulo em 15/08/2020 com boa recuperação. Teve diagnóstico incidental de adenocarcinoma pouco diferenciado na vesícula biliar de $1,5 \times 1,0 \mathrm{~cm}$ com invasão até camada adventícia, pT2bpN1. Invasão linfovascular e perineural positivas, com 1 linfonodo positivo de um examinado. Por conta do diagnóstico foi encaminhado ao centro especializado em câncer, Hospital A.C. Camargo - SP, para avaliação. Realizado PET/CT em 09/09/2020 sem alterações. Optou-se por não realizar complementação cirúrgica ou adjuvância por conta da coronariopatia grave. Em abril de 2021 apresentou aumento de CA19.9 (UI/L 649) e progressão de doença para fígado e tecido celular subcutâneo (região de trocater laparoscópico subxifoideano). Foi iniciado tratamento de primeira linha com Cisplatina e Gemzar com resposta de estabilidade radiológica da doença (redução do nódulo hepático e da lesão de subcutâneo) ao final do tratamento em novembro de 2021 e redução do CA19.9 para $162 \mathrm{UI} / \mathrm{L}$. O paciente foi encaminhado ao serviço de cirurgia abdominal com proposta de tratamento local da lesão abdominal em região epigástrica de $5 \mathrm{~cm}$, dolorosa, com ulceração e supuração de secreção serosa. O PET-CT de novembro de 2021 demonstrou área de concentração anômala do marcador 18F-FDG, correspondendo à lesão nodular sólida, de aspecto infiltrativo, nos planos mioadiposos da parede abdominal anterior à direita, na região do epigástrio, com SUV (Captação) de 6,7 (Figura 1). O paciente foi submetido à ressecção de lesão da parede abdominal com reconstrução miocutânea local e colocação de tela no defeito resultante no mesmo tempo cirúrgico (Figura 2). 
Apresentou boa evolução pós-operatória. Resultado anatomopatológico confirmou metástase de adenocarcinoma moderadamente diferenciado com invasão vasculolinfática e perineural presentes com margens livres. A lesão hepática foi previamente submetida a tratamento com radioterapia.

\section{Discussão}

Os casos apresentados tratam de CVB com desfechos diferentes. A primeira paciente operada no HSI, ainda na fase inicial de tumor e sem necessidade de reoperação ou tratamento adjuvante, e o segundo paciente operado em um hospital de São Paulo foi encaminhado ao A.C. Camargo, para orientação de tratamento da doença que já apresentava metástase linfonodal.

No caso da primeira paciente, com CVB diagnosticado por estudo histológico, após acompanhamento na clínica oncológica por 5 anos, não houve nenhum indício de recidiva da neoplasia. É importante salientar que além do risco de recidiva no sítio da colecistectomia, pode ocorrer disseminação intraperitoneal e portais, como ocorreu no caso encaminhado para tratamento

Figura 1. PET-CT do paciente operado no Hospital A.C. Camargo em São Paulo.

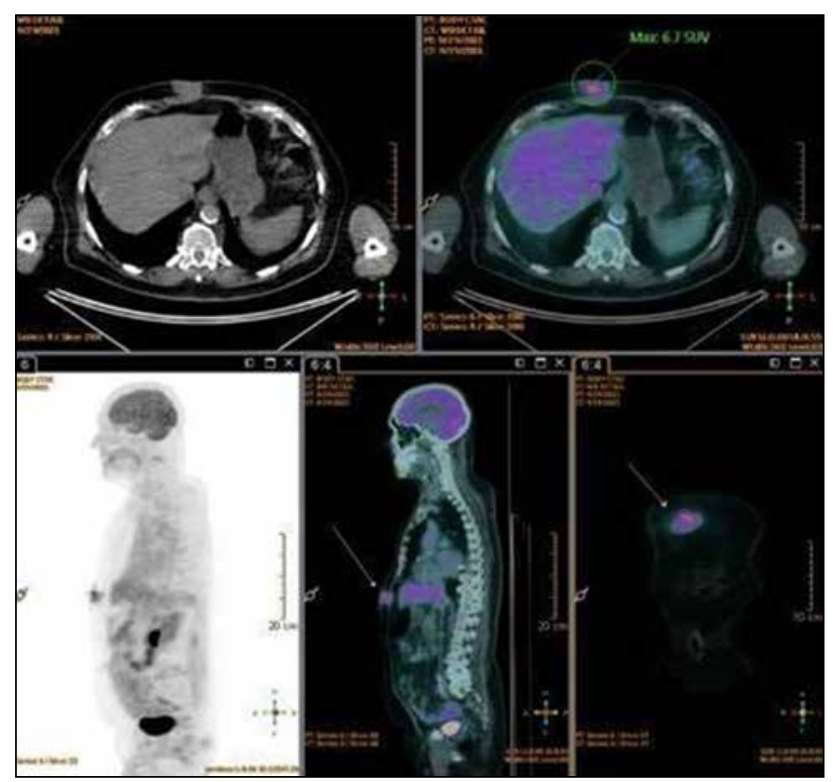

especializado no Hospital A.C. Camargo em São Paulo, motivo pelo qual as equipes de cirurgia do HSI e do A.C Camargo sempre retiram a vesícula biliar dentro de um envoltório previamente introduzido na cavidade peritoneal para esse fim.

Caso a paciente não tivesse sido submetida à colecistectomia, poderia ter um prognóstico reservado e provavelmente seria submetida a tratamento cirúrgico com a doença avançada, em detrimento das características inespecíficas do CVB, que muitas vezes só é diagnosticado em estágio avançado. Salientamos que pela falta de sintomas e resultados negativos nos marcadores oncológicos, não havia indicação para a paciente de utilização de métodos mais sofisticados como: ultrassonografia endoscópica, tomografia computadorizada e ressonância magnética. Entendemos que esses exames podem colaborar para um diagnóstico mais precoce do CVB. No presente caso, após a avaliação criteriosa da clínica oncológica e das características do tumor identificado no exame histopatológico, além da RM, optou-se pelo acompanhamento conservador rigoroso, a cada quatro meses, assim a reabordagem cirúrgica não foi indicada.

Figura 2. Fotografia tirada após ressecção de lesão da parede abdominal com reconstrução miocutânea local e colocação de tela.

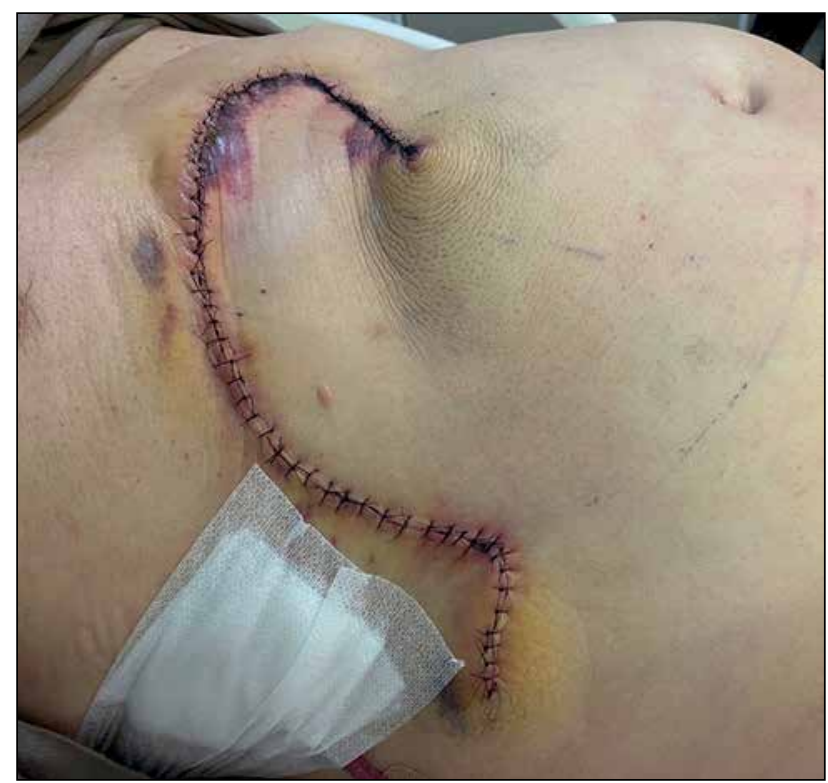


Para definir o tratamento da neoplasia de vesícula biliar, é necessário ter conhecimento do estadiamento do tumor, principalmente se é um tumor localizado ou se há metástases. Caso esteja localizado, o tratamento cirúrgico é o único capaz de oferecer a possibilidade de cura. Caso o tumor seja localmente avançado e não tenha metástases, o tratamento quimioterápico pode ser utilizado antes da cirurgia visando redução do tamanho da neoplasia. Outra possibilidade é o tratamento quimioterápico para controle da doença, como realizado no paciente de São Paulo (adjuvância).

Geralmente, nos casos diagnosticados no préoperatório, o tratamento cirúrgico preconizado é a colecistectomia radical, que envolve a retirada da VB juntamente com uma pequena parte do fígado adjacente (leito hepático) e linfonodos regionais. É preconizado nos casos de carcinoma incidental da vesícula biliar T1bN1 que seja feita complementação cirúrgica com hepatectomia dos segmentos 4 b e 5 ou ressecção do leito da vesícula até margens livres, associado a linfadenectomia regional. ${ }^{8}$ Tal procedimento não foi realizado no paciente de São Paulo pelo alto risco cirúrgico. Em alguns casos, pode ser necessária a realização de sessões de quimioterapia após a cirurgia (tratamento adjuvante). Caso a neoplasia tenha sofrido metástase, geralmente o tratamento cirúrgico não é indicado e a quimioterapia passa a ser a primeira escolha. ${ }^{9}$

\section{Conclusão}

O CVB é uma doença relativamente rara que tem difícil diagnóstico pré-operatório, o que pode levar os pacientes a terem mau prognóstico. Para tentar melhorar a abordagem desses casos é imperativo que as pesquisas identifiquem marcadores tumorais precoces e mais sensíveis. $\mathrm{Ou}$ ainda, que surjam novas tecnologias, nos exames de imagem, associadas ao aprimoramento dos meios disponíveis nos dias atuais, e que elas possam ser mais difundidas e utilizadas na prática médica diária. Assim, identificar e tratar de forma cada vez mais precoce e eficaz o $\mathrm{CVB}$, fornecendo longevidade e qualidade de vida, é o objetivo maior de quem trata esses pacientes.

\section{Referências}

1. Apodaca-Rueda, M., Cazzo, E., De-Carvalho, R. B., \& Chaim, E. A. (2017). Prevalência do câncer de vesícula biliar em pacientes submetidos à colecistectomia: experiência do Hospital de Clínicas da Faculdade de Ciências Médicas da Universidade Estadual de Campinas-UNICAMP. Revista do Colégio Brasileiro de Cirurgiões, 44, 252-256.

2. Randi, G., Franceschi, S., \& La Vecchia, C. (2006). Gallbladder cancer worldwide: geographical distribution and risk factors. International journal of cancer, 118(7), 1591-1602.

3. Laporte, G.A., Oliveira, A.F., Lucchese, A.M., Kalil, A.N., Silva, J.T.S., Santos, M.T.M. Diretrizes Oncológicas: Câncer de Vêsicula Biliar. Brasília: Diretrizes; 2018.

4. De Aretxabala X, Roa IS, Burgos LA, Araya JC, Villaseca MA, Silva JA. Curative resection in potentially resectable tumours of the gallbladder. Eur J Surg. 1997;163(6):419-26.

5. Hawkins WG, DeMatteo RP, Jarnagin WR, BenPorat L, Blumgart LH, Fong Y. Jaundice predicts advanced disease and early mortality in patients with gallbladder cancer. Ann Surg Oncol. 2004;11(3):3105.

6. Perpetuo MD, Valdivieso M, Heilbrun LK, Nelson RS, Connor T, Bodey GP. Natural history study of gallbladder cancer: a review of 36 years experience at M.D. Anderson Hospital and Tumor Institute. Cancer. 1978;42(1):330-5.

7. Apodaca-Rueda, M., Cazzo, E., De-Carvalho, R. B., \& Chaim, E. A. (2017). Prevalência do câncer de vesícula biliar em pacientes submetidos à colecistectomia: experiência do Hospital de Clínicas da Faculdade de Ciências Médicas da Universidade Estadual de Campinas-UNICAMP. Revista do Colégio Brasileiro de Cirurgiões, 44, 252-256.

8. Consenso brasileiro de carcinoma incidental de vesícula biliar. Arq Bras Cir Dig. 33 (1): e1496. Torres et. al, 2020.

9. Baiu I, Visser B. Gallbladder Cancer. JAMA. 2018;320(12):1294. 